\title{
A qualitative Study of TB Health Workers' and Stakeholders' Conceptualisation, Screening and Treatment of Mental Distress Experienced by TB Patients in Zambia
}

\section{Tila Mwinde Mainga ( $\sim$ Tila.Mainga1@lshtm.ac.uk)}

London School of Hygiene \& Tropical Medicine https://orcid.org/0000-0002-7711-3623

\section{Melleh Gondwe}

Zambart

\section{Robert C Stewart}

The University of Edinburgh Division of Psychiatry

Islay Mactaggart

London School of Hygiene \& Tropical Medicine

Kwame Shanaube

Zambart

\section{Helen Ayles}

London School of Hygiene and Tropical Medicine Faculty of Epidemiology and Population Health

\section{Virginia Bond}

London School of Hygiene and Tropical Medicine Faculty of Public Health and Policy

\section{Research}

Keywords: Tuberculosis, health workers, TB stakeholders, mental health, Treatment, Conceptualisation

Posted Date: March 17th, 2021

DOI: https://doi.org/10.21203/rs.3.rs-305063/v1

License: (c) (i) This work is licensed under a Creative Commons Attribution 4.0 International License. Read Full License 
A qualitative study of TB Health workers' and Stakeholders' Conceptualisation, Screening and Treatment of Mental Distress Experienced by TB Patients in Zambia

Author(s): T.Mainga ${ }^{1,2}$, M.Gondwe ${ }^{1}$, R.C. Stewart ${ }^{4,5}$, I.Mactaggart ${ }^{3}$, K.Shanaube ${ }^{1}$, H.Ayles ${ }^{1,3}$, V.Bond ${ }^{2,1}$

\section{Authors' information}

1.ZAMBART, University of Zambia School of Public Health, Ridgeway, Zambia

2. London School of Hygiene and Tropical Medicine, London, UK, Department of Global Health and Development

3. London School of Hygiene and Tropical Medicine, London, UK, Department of Department of Clinical Research

4. University of Edinburgh, Division of Psychiatry

5. Malawi Epidemiology and Intervention Research Unit (MEIRU), Lilongwe, Malawi

\section{Corresponding Author}

Tila Mainga

Global Health and Development

London School of Hygiene and Tropical Medicine

Email: Tila.Mainga1@lshtm.ac.uk; tila@zambart.org.zm

Word count (manuscript body): 5726 


\section{Abstract}

Background: There is an increased recognition to integrate mental health services into routine TB care. For a successful integration TB health workers and stakeholders need to be receptive to mental health services being delivered in tandem with TB services. Additionally, policy makers need to understand the current practices by TB health workers around treatment of mentally distressed TB patients. In this qualitative study we aimed to understand how TB health workers and other TB stakeholders viewed mental distress linked to TB and how they screened and treated mental distress in their patients.

Methods: The study draws on qualitative data collected in 2018 as part of the Tuberculosis Reduction through Expanded Antiretroviral Treatment and Screening for active TB trial (TREATS). TREATS was conducted in 8 urban communities in Zambia. The data was collected through 17 focus group discussions with local health committees $(n=96)$ and TB stakeholders $(n=57)$, and in-depth interviews with TB health workers $(n=9)$. Thematic analysis was conducted.

Results: TB stakeholders and health workers had an inadequate understanding of mental ill health and commonly described mental distress experienced by TB patients using the stigmatizing term "madness". Mental distress was also described as "overthinking", which participants attributed to psychosocial drivers of distress and inadequate knowledge of TB by TB patients rather than a condition that would benefit from a mental health intervention.

Conclusions: TB stakeholders and health care workers in Zambia are cognisant of the mental health implication of TB on TB patients. There are no standard screening or treatment options for mental distress in TB patients. TB health workers in Zambia could benefit from mental health training which should aim 
to increase knowledge about mental health and chronic illness while shifting negative attitudes around mental illness

Trial registration number: NCT03739736

Word Count: 290

Key Words: Tuberculosis, health workers, TB stakeholders, mental health, Treatment, Conceptualisation 


\section{Introduction}

2 Zambia has had a long-standing history with tuberculosis (TB) and efforts to eradicate it date back to the

3 1960s (1). Despite the major progress in relation to TB treatment and eradication, Zambia is still ranked

4 amongst the top thirty high TB burden countries globally (2). This in part is due to the high prevalence of

5 Human Immunodeficiency Virus (HIV) in the country. TB continues to be the most significant co-infection

6 for people living with HIV (PLWH) and cause of death for every 1 in 3 of this population (2). Zambia has an

7 estimated HIV prevalence of $11.3 \%$ amongst adults aged $15-49$ and an approximate HIV/TB coinfection

8 rate of $23.8 \%(3,4)$.

9 Poor mental health amongst TB patients acts as a barrier against TB eradication, by negatively impacting

10 health behaviours such as diet, health care seeking, medication adherence, and/or treatment completion

11 (5). TB and poor mental health not only share biological, social, and psychosocial risk factors but they also

12 exist in a syndemic relationship, acting synergistically to magnify the burden of disease and collectively

13 increase the risk for negative health outcomes (5). Similarly to other high TB burden settings, efforts to

14 eradicate TB in Zambia have not focused on the complex relationship between TB and poor mental health

$15(6,7)$. The lack of attention to mental health in TB could be attributed to the fact that the mental health

16 landscape in Zambia is still in its nascent phase with several service provision gaps including services being

17 highly centralized, with few trained mental health personnel $(8,9)$.

18 The literature points to varying prevalence rates of depression amongst TB patients. A global meta-

19 analysis that aimed to quantitatively summarize the prevalence of depression among TB patients found a

20 pooled estimate of $49.2 \%$ from 25 studies (10). 8 of 25 studies in the meta-analysis were from sub-

21 Saharan Africa, namely Nigeria, Cameroon, and Ethiopia, which like Zambia have high TB burdens. There

22 are a few studies looking at mental health and TB in the Zambian context, and these also highlight

23 potentially high levels of mental distress in TB patients, for example in a study conducted in 16 primary 
24 health centres in Zambia found $30.9 \%$ of 231 TB patients expressed suicidality with $3.4 \%$ of these having 25 attempted suicide at least once (11).

26 The 2015-2035 World Health Organization (WHO) End TB Strategy strongly advocates for the integration

27 of mental health and TB treatment (12), however low and middle income countries (LMIC) often lack the 28 trained mental health personnel needed to deliver such services (13). Sweetland et al. (13) highlight that 29 the task shifting model already established in TB care in LMICs, is well placed for the detection and 30 treatment of common mental disorders in TB patients. They emphasise that the bulk of TB care in LMICs, 31 including TB screening and follow up of participants, is conducted by community health workers, 32 therefore training this cadre of health workers in low-intensity psychological screening and treatment 33 would be an efficient way of integrating mental health service into the current TB infrastructure in LMICs 34 (13).

35 Health workers and TB stakeholders are at the core of TB eradication therefore their perspectives of the 36 mental health and TB nexus is integral to this initiative. Literature from the region highlights how negative 37 attitudes by health care workers serve as a barrier towards treatment for both TB and mental ill health 38 (14-16). To the best of our knowledge, there has been no work done to understand how TB stakeholders 39 and health workers in Zambia understand the mental health implications of TB on TB patients, and how 40 and if they currently navigate mental health screening and treatment in TB patients. We define TB 41 stakeholders as individuals or organisations that work directly with TB control or management. This study 42 sets out to explore the conceptualization of mental health and TB amongst TB health care providers and 43 stakeholders. The analysis also focuses on causes of mental distress in TB patients as understood by 44 health worker and stakeholders. Lastly, we also explore mental health screening and treatment practises 45 by TB health care providers in Zambia. 
47 The study draws on qualitative data collected in 2018 as part of the Tuberculosis Reduction through

48 Expanded Antiretroviral Treatment and Screening for active TB trial (TREATS). TREATS is a follow-on study

49 from the Population Effects of Antiretroviral Therapy to Reduce HIV Transmission (PopART) Trial which

50 was a randomised control trial that aimed to investigate the impact of combined community level TB/HIV

51 interventions on HIV incidence (17). PopART was conducted in 21 communities - 12 in Zambia and 9 in

52 South Africa.

53 The aim of TREATS is to measure the impact of the PopART intervention on TB incidence and prevalence.

54 TREATS is therefore being carried out in the same 12 PopART communities in Zambia. The TREATS

55 qualitative inquiry, where this data was collected, was conducted in 4 intervention and 4 control

56 communities. The qualitative work aimed to comparatively describe how TB patients experienced the

57 PopART intervention and document popular understanding of TB and TB stigma in these communities.

Data was collected by a social science team consisting of eight field-based social science graduates (4

males and 4 females) and three experienced social scientists who provided training, de-briefing and field support (first, second and last authors). Due to the sensitive nature of the data being collected researchers were trained to be sensitive to participants and reflective of their own biases towards mental health and

62 how their identity impacts participants and the resulting implications on the data collected.

63 In each of the eight communities, we conducted Focus Group Discussions (FGDs) with local health 64 committees Neighbourhood Health Committees (NHCs) and stakeholders working in the field of TB. This 65 method of data collection was selected to explore the perspectives of various stakeholders in the field of 66 TB. Locations most convenient for participants were organised prior to the discussions, and venues were 67 chosen to maximise privacy of participants. Open ended questions guided by a semi structured interview guide were asked to encourage wide-ranging perspectives. Each FGDs was facilitated by a moderator who 69 guided the discussion and an assistant moderator who assisted with facilitation and note taking. Each FGDs was approximately 1.5 to 2 hours long. 
71 Data was also collected from In-depth Interviews (IDIs) with TB health workers. IDIs were chosen due their

72 ability to provide rich contextual evidence on mental health conceptualisation, and experiences of mental

73 health treatment and management of TB patients by TB health care workers. Interviews lasted between

7445 mins to 1.5 hours. Interviews were conducted in a private and comfortable location for the

75 participants. A semi structured interview guide was used with probes where appropriate.

77 FGDs and IDIs interview guides included mental health questions which were guided by the literature.

78 Areas of inquiry included the understanding of mental health in the Zambian context, the causes of mental

79 distress during TB diagnosis and treatment, the implications of poor mental health for TB patients' quality

80 of life and TB treatment outcomes, and availability and accessibility of mental health services for TB

81 patients.

82

\section{Recruitment}

84 The FGDs were conducted with a maximum of 10 to 12 participants. Maximum variation sampling was

85 employed, with a spread based on age, sex, and occupation. Participants were chosen with the aim of

86 encouraging varied viewpoints while keeping the atmosphere and power relations comfortable enough

87 for everyone to contribute. Purposive sampling was used to recruit health care workers for the IDIs. The

88 eligibility criteria for health care workers and the NHC participants included providing key TB services in

89 the clinics from the eight TREATS communities during the time of the PopART trial through data collection

90 for this study. Eight of the health care workers recruited were nurses and one was a health technician

91 who all worked predominantly with diagnosed TB patients by providing medication and follow up care in

92 their respective clinics. NHC members were recruited through the help of the community mobilisers, who

93 serve as the link between the TREATS study and the community members. Clinic-based TB treatment 
94 supporters were also used to help identify members of the NHC. Subsequently the TB stakeholders in each

95 community were identified through FGDs with the NHC. TB stakeholders recruited included government

96 organizations such as schools and clinics and non-government organizations such as churches, public

97 health institutions, home based care organizations. All stakeholders provided different types of TB

98 services including screening, testing and follow up care for individuals with TB in their respective

99 communities. Table 1 provides a summary of data collection activities and description of participants.

100

101

Table 1: Table of Participants

\begin{tabular}{|l|l|l|l|}
\hline Activity & Participant Type & \multicolumn{2}{l|}{ Characteristic } \\
\hline In-depth interviews & TB Health Worker (N=9) & Male & 2 \\
\cline { 2 - 3 } & & Female & 7 \\
\hline Focus Group Discussion & NHC (N=96) & Age Range & $25-58$ \\
\hline & Stakeholders (N=57) & Number of activities & 9 \\
\hline
\end{tabular}

102

103

\section{Data Analysis}

105 IDIs and FGDs were recorded and transcribed verbatim. Data was analysed thematically using a coding

106 framework developed around the TB mental health literature. Further codes were added during the

107 analysis. Data was double coded by the first and second authors using ATLAS ti software. Any 
discrepancies with themes emerging from the data were resolved with correspondence with the last author and securing common consent among the first, second and last authors.

\section{Results}

The results are presented under three broad themes. The first explores TB stakeholders' and health care providers' conceptualization of mental health with regards to TB patients. The second explores causes of mental distress in TB patients as understood by TB stakeholders and health workers. Thirdly, the results explore how TB health workers screen and treat mental distress in TB patients.

\section{Conceptualisation of Mental health by TB health workers and stakeholders}

The term 'mental health' did not illicit definitions from health workers and TB stakeholders that were equivalent to a biomedical definition of the term. Rather, "Madness" (Kufunta) was the most common definition of mental illness stated by stakeholders, a definition with strong stigmatizing undertones towards the mentally ill. For example, when asked to define mental health, one NHC FGD (Z3) said it was "madness of the mind".

There was a belief among some stakeholders that TB causes "madness" due to physiological factors such the "TB bacteria entering the brain" of the patient or side effects of the anti-TB medication.

"It depends on the area that the TB germ goes to live in the body. If its TB of the brain, it can make the person mad, they would be looking mad." (FGD, NHC, Z3)

The symptoms of 'madness' provided by stakeholders included: patients behaving irrationally, being short tempered and aggressive, hoarding items such as stones and animal skins, refusing to take their 
medication, "delusional behaviour", extreme sex drive (particularly in men) or no sex drive at all, and memory loss.

When asked specifically about depression and anxiety disorders in TB patients, stakeholders and health workers had different ways of expressing these conditions. Some of the definitions included TB patients being "over emotional, emotionally stressed or emotional," but most commonly they were described as "overthinking or thinking too much." ("Ku ganiza maningi") by most of the participants. Participants described TB patients who were over thinking or thinking too much as being overly sad, and incessantly worried with a tendency of isolating themselves. Most participants acknowledged that this state of mind frame was very common in TB patients especially upon hearing their diagnosis. Only a handful of participants referred to depression and anxiety by their clinical names when describing the symptoms in TB patients.

"They (TB patients) suffer-they suffer from depression, anxiety... it can even lead them to paralysis, where the person refuses to even get up, the person just says ' uhh no, I can't do this'.." (FGD, TB Stakeholder, Z12)

\section{Causes of mental distress in TB patients as understood by stakeholders and health care workers.}

According to TB stakeholders and healthcare workers there are three main causes of mental distress in TB patients: loss of income resulting from TB morbidity, TB stigma, and misinformation or lack of adequate information about TB transmission and treatment compounded by a fear of death.

\section{Loss of ability to earn a living.}

Participants highlighted that TB diagnosis often had significant economic implications on TB patients and this was a source of mental distress for many. They stressed that TB patients often worked in the informal sector and therefore their ability to earn was dependant on their ability to work. Participants highlighted 
153 that few of their patients had the liberty of paid sick leave. The stakeholder below details their experiences

154 with some TB patients regarding how their TB diagnosis could affect their income and the ripple on 155 implication this loss of income has on the patient's family and ultimately the patient's mental health.

"He (TB patient) is the one who goes for work, right, then he is diagnosed with $T B$, and he is told that he can't go for work, you see these jobs that they (TB patients) do, unless they report for work they will not get paid, if they don't report for work they won't be paid. So, this adult will be thinking a lot...how I am going to feed my children. If it means going to the fields to farm, he is not able to because of the disease, so he will have a lot of thoughts about how to look after the family while he is sick. So, they have double problems, planning for the family and looking after themselves while they have the disease......that's where I see agony to be biggest. (FGD, NHC, Z12)

$\underline{\text { Stigma }}$

Most stakeholders and health care workers believed that TB stigma was a major driver of mental ill health in TB patients. One stakeholder described stigmatization as "mental torture" that would eventually result

167 in "madness" in TB patients. The participant below went as far as to state that in the absence of stigma, 168 TB patients would not develop depression.

"If they (TB patient) are not being stigmatized they can't go through depression" (FGD, NHC, Z4)

170 The stigma that most participants alluded to was at household level, namely family members or close

171 friends that live with the TB patients. The stigma was mainly in form of forced isolation, for example 172 participants highlighted that some care givers make TB patients use their own eating utensils, beddings, 173 or sleep in separate rooms. 
"Another thing that contributes (to mental distress) is how those (family members) with TB patients behave. They avoid sitting near them (TB patients) ...... Even eating with TB patients, they refuse saying, 'you might contract TB'.' (FGD, NHC, Z7)

Another participant in the same FGD went on to share a personal experience of how such behaviours led to their brother contemplating suicide whilst on TB treatment.

"I have a younger sibling his name is X, he had TB. When he had TB I used to stay with him and my in laws..., my aunt used to treat my brother in a way similar to what we discussed, where she would give him his own cup, plate and his own room.., he used to have worries because of this treatment. He reached an extent of saying I would rather hang myself instead of me staying alone, there is no one close to me, no one even chats with me..."(FGD, NHC, Z7)

Aside from stigma in the household, participants brought to light the fact that TB patients experience stigma in the health care setting as well, particularly from health care workers providing non-TB services. The negative attitudes towards TB patients by health workers were attributed to fear of contracting TB and inadequate training about TB in general. Stigmatizing behaviours towards TB patients by health workers included talking to TB patients rudely or denying them health services as seen in the quote below. R: The pharmacist said "no no no, he (TB patient) is infectious he will infect others so you should not be allowing him to come here he should just stay at the TB corner. The peers should come and collect his drugs on his behalf...." that is what happened

I: So do health workers stigmatise TB patients?

R: They stigmatised that one 


\section{$201 \quad$ Misinformation about TB}

202 Another common driver of mental distress amongst TB patients cited by participants included a lack of understanding of TB transmission and the fact that TB is curable with adequate treatment.

"I think it (mental distress) all goes back to lack of knowledge and understanding of the TB infection. To some people, once they are diagnosed with TB, they will feel emotionally defeated

Participants also highlighted the mental health repercussions brought about by the idea that TB is incurable and how this misinformation was often led to a fear of death. won't get well and they will die". (FGD, TB Stakeholder, Z3)

213 This lack of understanding about TB treatment highlighted by stakeholders and health care workers was

214 reflected in their experiences with TB patients who explained their TB infection with popular community 215 beliefs about TB. According to participants, patients mentioned beliefs that TB was contracted through 216 excessive drinking or smoking, 'promiscuous' behaviour (having sex with multiple partners) or sexual 217 contact with a woman who had had an abortion. These popularly held beliefs served as drivers of TB 218 stigma, increased feelings of shame, and reduced self-worth amongst TB patients. Furthermore, 
participants stressed that these beliefs made it harder for TB patients to understand how they were

220 infected with TB as illustrated by the stakeholders experience below.

"After seeing her TB results in red pen stating smear positive and being told that she was found with $T B$ and had to start drugs, she started crying saying "I don't sleep with men, am not a prostitute how can I be found with TB ....," (FGD, TB Stakeholder, Z8)

Additionally, participants noted that TB patients often associated TB with HIV, with many of their TB patients automatically assuming that their TB diagnosis meant that they were also HIV positive. According to participants this association between the two conditions was often emotionally distressing for TB 227 patients.

Others had beliefs to say when you have TB, and definitely when you start TB drugs, then you will have HIV, those were the misconceptions that they had...TB patients think that when they start taking TB drugs people will know that they are HIV positive (FGD, TB Stakeholder, Z8)

Participants did note, however, that in reality a TB and HIV co-morbidity did present an extra emotional burden on TB patients. TB health workers bore witness to how emotionally challenging it was to accept and cope with having TB and HIV. For some of their patients the co-morbidity, they felt, underscored experiences of denial and depression and could lead to death. "They (two TB patients) were depressed because they were told that they had TB and HIV. The one who was from area $X$ was very much in denial. He refused to believe he could have TB or HIV because he claimed he never had sex with women. That is how he died. Then the other one had a wife who was pregnant. He also refused to accept that he had TB. A lot of them believe when you have TB then you automatically have HIV. He refused to accept it. We tried by all means to help and counsel him. When you are around, he would accept to come (to the clinic) but when you leave his home, he would change his mind. That is how he died". (FGD, NHC, Z2) 
243 TB health workers and stakeholders expressed that mental health screening is not part of routine TB 244 treatment in Zambia. Both diagnosis and treatment of mental illness were reliant on the health workers' 245 interpretation of patients' emotional state. For example, some health care workers said they would talk 246 to specific patients if the patient looked visibly upset by the news of their TB diagnosis or appeared to be 247 isolating themselves too much from others. themselves and ask questions where they are not sure. We then explain to them according to the questions they are asking. We give them time to talk and ask questions". (IDI, Health Care Worker, Female, unknown age, Z8).

Most health care workers interviewed stated that they had not received any in-depth training regarding mental health. Some had undergone short psycho-social counselling courses but even such courses were not targeted towards handling chronically ill patients with depression and anxiety. Moreover, health workers rarely received refresher trainings on mental health. Limitations in mental health training of TB 257 healthcare workers was reflected by TB stakeholders. with depression. They are trained in general counselling. Where they talk on the surface and try to calm the person (distressed patient). We don't have someone who's trained specifically for depression or organizations that have come in to help on matters of depression." (FGD, TB stakeholder, Z3) 
264 The treatment offered for mental distress was often a discussion around TB treatment with the belief that

265 all that distressed patients needed was encouragement and knowledge about their condition. Most of

266 these talks were provided by nurses in charge of TB treatment and TB treatment supporters. TB treatment

267 supporters also provided educational talks informing patients and the community about how TB is spread,

268 and how to take care of themselves while they have TB. These health talks place a lot of emphasis on the

269 importance of adherence to medication to get cured from TB and often try to dispel misconceptions about

270 TB.

"..... Every time they (TB patients) take their drugs we make them sit here and then we ask 'what is your problem? Then we try to explain what TB is and give them information about TB. We ask the patient, 'do you really understand this disease you have?', 'where you contracted it?', 'how do you feel?', 'where do you feel you got it?' and they will try to explain to us and then we chip in and give the information if there are any myths and misconceptions." (IDI, Health Care Worker, Female, Unknown age, Z8)

278 example when asked about emotional distress in her patients one nurse simply stated "Hmm, I am not a mental nurse... I don't have a psychiatric paper (degree)." IDI, Health Care Worker, Z7. Following this line 280 of thought some health workers and stakeholders opted to refer emotionally distressed patients to the 281 country's main psychiatric hospital. “.....at times we refer those people (TB patients), in case, it becomes complicated, like when they start becoming mentally ill...."(FGD, TB stakeholders, Z7)

\section{Discussion}


TB stakeholders and health care providers conceptualization of mental ill health in TB patients was

287 categorised in two distinct concepts, the first being madness depicted by behavioural and cognitive

288 symptoms similar to those seen in western conceptualization of psychotic disorders. Madness in TB patients was attributed to physiological factors such as TB entering the brain of TB patients or a side effect of TB medication. The second description of mental ill health was a state where TB patients think too much or are overthinking. According to participants this state of mind in TB patients was brought about by more social than biological factors such as loss of income attributed to TB, experiences of TB stigma, and lack of adequate information about TB infection and treatment. Participants described TB patients who were overthinking or thinking too much with symptoms that had striking overlaps with depression and anxiety disorders. Examples include TB patients being very worried and overly sad with behaviours such as isolation or suicidal ideation. They noted the negative implications that this state of mind had on TB patients' overall wellbeing. Participants believed that providing TB patients with enough information about TB infection and treatment should be sufficient to allow TB patients to overcome this state of mind. The data for this study was organised and interpreted through a holistic exploration of mental health during TB treatment from perspectives of TB stakeholders and health care providers.

"Madness" (Kufunta) was the primary way in which TB stakeholders and health worker participants described the mental health and potential mental illness in TB patients. Indeed there is some literature that suggests TB infection (5) and anti-TB medication can lead to neuropsychiatric complications in TB patients, however these complications are rare as depicted in a global meta-analysis that calculated a pooled estimate of $1.1 \%(95 \% \mathrm{Cl} 0.2-2.1)$ for central nervous system related adverse drug reactions (18).

307 However, our findings reveal that this conceptualisation of mental illness was inundated with stigmatizing undertones as participants described mentally ill TB patients with disparaging terms such as "disorganised, 
number of studies done on the attitudes towards mental illness in Zambia $(9,14,19,20)$. For example,

311 Kapungwe et al. (14) indicate that stigma towards mental illness was pervasive, even amongst primary

312 health care providers and across different levels of society in Zambia. They further highlight that it is an

313 all-embracing form of stigma as it permeates across those labelled as mentally ill and extends to their

314 family members and even to health institutions where mentally ill patients receive treatment. Our

315 researchers were not impervious to these attitudes. Despite comprehensive training in mental health

316 prior to data collection, research assistants still used stigmatizing language such as "madness, and sickness

317 of the mind" in reference to mental ill health when conducting interviews. This highlights how deep-

318 rooted mental health biases are in this context and the potential challenges that may arise in attitudinal

319 shifts towards mental illness.

320 These attitudes towards mental illness are present even at policy level, evidenced by the $1951-2019$

321 Mental Disorders Act use of terms such as 'idiots, imbaciles, and invalids' in reference to patients with

322 mental illness (8). The historical gaps with mental health service provision in Zambia could be partly

323 addressed by a recent Mental Health Act passed in April 2019. Among other things, the Act aims to

324 increase awareness of mental health and tackle mental health stigma (21). Implementation of these

325 changes (which will require sustained government commitment and increased funding allocation) could

326 potentially improve the mental health services available to TB patients.

327 The stigmatizing attitudes of mental illness are not unique to Zambia but are prevalent through the region

328 (22-24).They are, in most part, driven by traditional beliefs in supernatural causes of mental illness such

329 as witchcraft $(19,24)$. In our findings however, TB occupied a parallel space to witchcraft, as it provided

330 explanations to the causes of 'madness' in TB patients. This mirrors findings from work focused

331 conceptualisation of mental health among people living with HIV in the region (25). 
332 There are profound negative implications of these stigmatizing attitudes for TB patients dealing with 333 mental ill health on both their quality of life and health seeking behaviours (26). TB alone, is a highly 334 stigmatized condition in Zambia (35), furthermore TB is strongly associated with HIV which is also 335 stigmatised in this context (27). Therefore, TB patients suffering from mental distress could experience a 336 convergence of multiple stigmas. It is therefore vital to tackle the multiple stigmas associated with mental 337 ill health, TB and other stigmatized conditions that people may be living with. The health-related stigma 338 framework lends itself well as a guiding tool to tackling stigma brought about by mental ill health and TB 339 as it acknowledges and addresses parallels in drivers and consequences of stigma across different 340 conditions and recommends a cross-cutting approach to addressing stigma (38).

341 Aside from madness, TB health workers and stakeholders also described TB patients in mental distress as 342 overthinking/thinking too much, a description similar to other countries in the region (28-31). Our 343 participants' conceptualization of overthinking/thinking too much, has overlaps with depression, for 344 example, both are characterised by sadness/low mood, isolation, and suicidal ideation. However, there 345 are significant differences between the two, namely, overthinking/thinking too much, unlike depression, 346 was not considered to be an actual illness by participants and can be alleviated through provision of 347 adequate information about TB and rendering encouragement to TB patients who appear distressed. The 348 conceptualisation of overthinking/thinking too much as a social problem, which consequently requires a 349 social solution is echoed in work from the region. For example, in work with HIV positive women in South 350 Africa, HIV infection was not considered to be the cause of their mental distress but rather social factors 351 such as stigma, poverty, and stressful life events resulting from their condition were to blame. Accordingly 352 social support emerged as the most appropriate intervention for mental distress by the majority of 353 participants in this study (32). Psychosocial interventions are non-pharmacological and are focused on 354 psychological or social factors of distress. Psychosocial interventions are shown to be effective in treating 355 mental disorders such as depression in physically ill individuals, as seen in a systematic review and meta- 
analysis which concluded that psychosocial interventions, such as therapy, have a beneficial effect for

357 PLWH with mental health problems (33).

358 In context of these findings, it is worth addressing the social underlying causes of mental distress in TB 359 patients including improving social security nets for economically vulnerable TB patients, and as noted 360 above, addressing TB, mental health and HIV stigma. These stigma reduction initiatives should also aim to 361 dispel misconceptions about TB while providing accurate information about TB infection and treatment 362 in a context appropriate manner.

363 Viewing nonpsychotic mental illness, exclusively as a social phenomenon potentially dismisses the 364 biomedical aspects of mental illnesses. Therefore, TB patients in need of biomedical support with handling 365 their mental distress are at risk of being overlooked. This is a particular concern considering our findings 366 indicate that there are no standard screening and diagnosing mechanisms for TB patients suspected to be 367 dealing with mental distress in Zambia. Both the diagnosis and consequent treatment options were reliant 368 on health workers' perceptions of the patient. Additionally, it is worth noting that this lack of adequate 369 mental health screening and treatment is in a context with a potentially high prevalence of common 370 mental disorders (13\%) and this prevalence is estimated to be twice as high in individuals living with 371 chronic illness such as HIV (21\%) (34).

372 Zambia is extremely under resourced in relation to trained mental health personnel (8). As Sweetland et 373 al, (13) argue, a context such as Zambia could lend itself well to the WHO Mental Health Gap Action 374 Programme (mhGAP) model. mhGAP trains non health specialists in detection and management of ten 375 priority disorders including psychosis, alcohol and drug use, depression, and suicide (35). This model 376 places emphasis on pharmacological treatments while providing some brief structured psychotherapies 377 that can be delivered by non-specialists (35). In the Zambian context such training should be provided to 378 both clinic-based health care providers and community health workers, who are at the helm of TB 
management. Community health workers provide services such as community TB screening, contact

380 tracing and follow up and monitoring of TB patients in the community. Community health workers

381 established position within local communities and the primary care structure would allow for a more

382 contextually sensitive understanding and delivery of mental health services, an understanding that may

383 be able to walk the delicate line between psychosocial and biomedical aspects of mental health.

384 The model of training nonspecialised health workers in detection of mental disorders has proved to be 385 somewhat effective in the region. For example, knowledge about mental health disorders and confidence 386 in identifying mental health disorders in both clinic based (36-38) and community based (39) health care 387 workers have been found to improve significantly after a mental health training intervention. There are 388 obvious concerns with this approach including a potential over use of pharmacological solutions to 389 address psychosocial challenges, resource constraints affecting adequate supervision of non-specialised 390 mental health staff (40) and lastly the need to adequately motivate and compensate community health 391 workers without relegating their dedication and constantly evolving roles and responsibilities to bouts 392 of altruism (41). Furthermore, if such an initiative is adopted it would need to be adequately evaluated to 393 both inform areas of improvement and adoption in similar context (42).

394 These findings come at a timely juncture, as the world of TB contemplates ways of integrating mental 395 health screening and treatment into the TB. The work provides insight into how health workers and 396 stakeholders working in the field of TB understand the intersection between mental health and TB in the 397 Zambian setting, thereby highlights baseline knowledge and gaps that policy makers need to consider as 398 TB programs aim to integrate mental health care in their services.

\section{Limitations}

Despite training, some research assistants in the study still had inherent biases towards mental health,

401 which were present in their interpretation/communication of the mental health questions to participants. 
402 The major strengths of the study were the large sample size and geographical reach of participants which

403 spanned across 8 urban communities in Zambia, this allowed for exploration of diverse experiences and 404 views around mental health and TB.

405 Conclusion

406 TB stakeholders and health care workers are cognisant of the mental health implication of TB on TB 407 patients, albeit understanding mental health through an either stigmatizing lens or one that is overly 408 reliant on the psychosocial drivers of the distress. Health workers generally lack the toolkit to adequately 409 detect and treat mental disorders in their patients and could therefore benefit from mental health

410 training. The mental health training and sensitization for health care workers should aim to increase 411 knowledge about mental health and chronic illness while shifting negative attitudes around mental illness.

412 There is also a need for standardised pathways for recognition and treatment of TB patients who appear 413 to be mentally distressed or ill. Lastly social drivers of mental distress such as TB stigma and economic 414 vulnerability also need to be addressed to help alleviate mental distress in TB patients. Ethics approval and consent to participate

417 Ethical approval for all study procedures was obtained from the institutional review of the London School 418 of Hygiene and Tropical Medicine (LSHTM) (\#14985), and the Bio-medical Ethics Committee of the 419 University of Zambia (005/02/18). Written consent was obtained from all participants prior to data 420 collection activities.

423 NCT03739736- Registered on the 14th November 2018- Retrospectively registered- 
424 https://clinicaltrials.gov/ct2/results?cond=\&term=NCT03739736\&cntry=\&state=\&city=\&dist

425

426 Consent for publication

427 Consent for publication was obtained from all participants, and consent forms would be available on

428 request.

429

430 Availability of data and materials

431 The data used or analysed during the current study are available from the corresponding author on

432 reasonable request.

433

434 Competing Interests

435 This project is part of the EDCTP2 programme supported by the European Union (grant number RIA2016S-

436 1632-TREATS). The views and opinions of authors expressed herein do not necessarily state or reflect

437 those of EDCTP. The authors have no competing interest to declare

438

$439 \quad$ Funding

440 This research is funded by the EDCTP2 programme supported by the European Union (grant number

441 RIA2016S-1632-TREATS).

442

443 Authors' contributions 
$444 \mathrm{TM}, \mathrm{MG}, \mathrm{VB}$, designed the study. MG managed data collection teams with oversight from TM and VB. TM, 445 MG and VB collected some of the data. TM and MG coded the data, TM led the analysis with input from

446 VB. TM wrote the first draft of the manuscript which was edited by VB. TM, VB, IM, RS, MG KS AND HA, 447 contributed to the interpretation of the findings and commented on the drafted manuscript. All authors 448 read and approved the final manuscript.

450 Acknowledgements

451 We are grateful to all the participants for giving their time and participating willing. We are also grateful 452 to the TREATS project Publication Working Group for their edits.

454 Authors' information (optional)

455 1.ZAMBART, University of Zambia School of Public Health, Ridgeway, Zambia

456 2. London School of Hygiene and Tropical Medicine, London, UK, Department of Global Health and 457 Development

458 3. London School of Hygiene and Tropical Medicine, London, UK, Department of Department of Clinical 459 Research

$460 \quad 4$. University of Edinburgh, Division of Psychiatry

461 Abbreviations

462 FGDs: Focus Group Discussions

463 IDIs: In-depth Interviews 
464 HIV: Human Immunodeficiency Virus

465 LMIC: Low- and Middle-Income Countries

466 LSHTM:London School of Hygiene and Tropical Medicine

467 MhGAP: Mental Health Gap Action Programme

468 NHCs: Neighbourhood Health Committees

469 PLWH: People Living with HIV

470 PopART: Population Effects of Antiretroviral Therapy to Reduce HIV Transmission

471 TB: Tuberculosis

472 TREATS: Tuberculosis Reduction through Expanded Antiretroviral Treatment and Screening for active TB

473 trial

474 WHO: World Health Organization

\section{Bibliography}

476 1. Mwaba P, Maboshe M, Chintu C, Squire B, Nyirenda S, Sunkutu R, et al. The relentless spread of

477 tuberculosis in Zambia-trends over the past 37 years (1964-2000). South African Medical Journal.

$478 \quad 2003 ; 93(2): 149-52$.

$4792 . \quad$ World Health Organization. World Health Organization - 2019 - Global tuberculosis report 2019.

4802019.

481 3. Chanda-Kapata P, Kapata N, Klinkenberg E, Grobusch MP, Cobelens F. The prevalence of HIV

482 among adults with pulmonary TB at a population level in Zambia. BMC Infect Dis. 2017;17(1):236.

483 4. Mudenda SS, Kamocha S, Mswia R, Conkling M, Sikanyiti P, Potter D, et al. Feasibility of using a

484 World Health Organization-standard methodology for Sample Vital Registration with Verbal Autopsy 
485 (SAVVY) to report leading causes of death in Zambia: results of a pilot in four provinces, 2010. Popul

486 Health Metr. 2011;9:40.

487 5. Sweetland AC, Kritski A, Oquendo MA, Sublette ME, Norcini Pala A, Silva LRB, et al. Addressing 488 the tuberculosis-depression syndemic to end the tuberculosis epidemic. Int J Tuberc Lung Dis.

$489 \quad 2017 ; 21(8): 852-61$.

490 6. Ambaw F, Mayston R, Hanlon C, Alem A. Depression among patients with tuberculosis:

491 determinants, course and impact on pathways to care and treatment outcomes in a primary care setting

492 in southern Ethiopia--a study protocol. BMJ Open. 2015;5(7):e007653.

493 7. Issa BA, Yussuf AD, Kuranga SI. Depression comorbidity among patients with tuberculosis in a 494 university teaching hospital outpatient clinic in Nigeria. Mental health in family medicine. 2009;6(3):133.

$495 \quad$ 8. Ngungu J, Beezhold J. Mental health in Zambia-challenges and way forward. International 496 Psychiatry. 2009;6(2):39-40.

497 9. Mwape L, Sikwese A, Kapungwe A, Mwanza J, Flisher A, Lund C, et al. Integrating mental health 498 into primary health care in Zambia: a care provider's perspective. International Journal of Mental Health 499 Systems. 2010;4:21.

500 10. Duko B, Bedaso A, Ayano G. The prevalence of depression among patients with tuberculosis: a 501 systematic review and meta-analysis. Annals of General Psychiatry. 2020;19:1-11.

502 11. van den Heuvel L, Chishinga N, Kinyanda E, Weiss H, Patel V, Ayles H, et al. Frequency and 503 correlates of anxiety and mood disorders among TB- and HIV-infected Zambians. AIDS Care.

$504 \quad 2013 ; 25(12): 1527-35$.

505 12. World Health Organization. Implementing the end TB strategy: the essentials. World Health 506 Organization; 2015. Report No.: 9241509937. 
13. Sweetland AC, Jaramillo E, Wainberg ML, Chowdhary N, Oquendo MA, Medina-Marino A, et al.

508 Tuberculosis: an opportunity to integrate mental health services in primary care in low-resource

509 settings. The Lancet Psychiatry. 2018;5(12):952-4.

510 14. Kapungwe A, Cooper S, Mayeya J, Mwanza J, Mwape L, Sikwese A, et al. Attitudes of primary

511 health care providers towards people with mental illness: evidence from two districts in Zambia. Afr J

$512 \quad$ Psychiatry (Johannesbg). 2011;14(4):290-7.

513 15. Phetlhu DR, Watson MJ. Perceptions and attitudes of health workers towards patients co-

514 infected with HIV and tuberculosis. Journal of Social Sciences. 2011;29(1):23-7.

515 16. Ibrahim LM, Hadjia IS, Nguku P, Waziri NE, Akhimien MO, Patrobas P, et al. Health care workers'

516 knowledge and attitude towards TB patients under Direct Observation of Treatment in Plateau state

517 Nigeria, 2011. The Pan African Medical Journal. 2014;18(Suppl 1).

518 17. Hayes RJ, Donnell D, Floyd S, Mandla N, Bwalya J, Sabapathy K, et al. Effect of Universal Testing

519 and Treatment on HIV Incidence - HPTN 071 (PopART). N Engl J Med. 2019;381(3):207-18.

520 18. Hwang TJ, Wares DF, Jafarov A, Jakubowiak W, Nunn P, Keshavjee S. Safety of cycloserine and

521 terizidone for the treatment of drug-resistant tuberculosis: a meta-analysis. Int J Tuberc Lung Dis.

$522 \quad 2013 ; 17(10): 1257-66$.

523 19. Kapungwe A, Cooper S, Mwanza J, Mwape L, Sikwese A, Kakuma R, et al. Mental illness-stigma

524 and discrimination in Zambia. African Journal of Psychiatry. 2010;13(3).

525 20. Munakampe MN. Strengthening mental health systems in Zambia. International Journal of

526 Mental Health Systems. 2020;14(1):28.

527 21. Ministry of Health GoZ. The Mental Health Act No. 6 of 2019 MoH, Goverment of Zambia;

5282019.

529 22. Egbe CO, Brooke-Sumner C, Kathree T, Selohilwe O, Thornicroft G, Petersen I. Psychiatric stigma

530 and discrimination in South Africa: perspectives from key stakeholders. BMC Psychiatry. 2014;14(1):191. 
531 23. Tawiah PE, Adongo PB, Aikins M. Mental Health-Related Stigma and Discrimination in Ghana:

532 Experience of Patients and Their Caregivers. Ghana Med J. 2015;49(1):30-6.

533 24. Patel V, Mutambirwa J, Nhiwatiwa S. Stressed, depressed, or bewitched? Development in 534 Practice. 1995;5(3):216-24.

535 25. Mayston R, Frissa S, Tekola B, Hanlon C, Prince M, Fekadu A. Explanatory models of depression 536 in sub-Saharan Africa: Synthesis of qualitative evidence. Social Science \& Medicine. 2020;246:112760.

537 26. Pachi A, Bratis D, Moussas G, Tselebis A. Psychiatric morbidity and other factors affecting

538 treatment adherence in pulmonary tuberculosis patients. Tuberc Res Treat. 2013;2013:489865.

539 27. Hargreaves JR, Krishnaratne S, Mathema H, Lilleston PS, Sievwright K, Mandla N, et al. Individual 540 and community-level risk factors for HIV stigma in 21 Zambian and South African communities: analysis

541 of data from the HPTN071 (PopART) study. AIDS. 2018;32(6):783-93.

542 28. Chibanda D, Mesu P, Kajawu L, Cowan F, Araya R, Abas MA. Problem-solving therapy for 543 depression and common mental disorders in Zimbabwe: piloting a task-shifting primary mental health 544 care intervention in a population with a high prevalence of people living with HIV. BMC public health. $545 \quad 2011 ; 11(1): 828$

546 29. Johnson LR, Mayanja MK, Bangirana P, Kizito S. Contrasting concepts of depression in Uganda:

547 Implications for service delivery in a multicultural context. American Journal of Orthopsychiatry.

$548 \quad 2009 ; 79(2): 275-89$.

549 30. Familiar I, Sharma S, Ndayisaba H, Munyentwari N, Sibomana S, Bass JK. Community perceptions 550 of mental distress in a post-conflict setting: A qualitative study in Burundi. Global public health.

$551 \quad 2013 ; 8(8): 943-57$.

552 31. Okello ES, Ekblad S. Lay concepts of depression among the Baganda of Uganda: a pilot study. 553 Transcultural psychiatry. 2006;43(2):287-313. 
554 32. Petersen I, Hancock JH, Bhana A, Govender K. Closing the treatment gap for depression co-

555 morbid with HIV in South Africa: Voices of afflicted women. 2013.

556 33. van Luenen S, Garnefski N, Spinhoven P, Spaan P, Dusseldorp E, Kraaij V. The Benefits of

557 Psychosocial Interventions for Mental Health in People Living with HIV: A Systematic Review and Meta-

558 analysis. AIDS Behav. 2018;22(1):9-42.

559 34. Chipimo PJ, Fylkesnes K. Mental distress in the general population in Zambia: impact of HIV and 560 social factors. BMC Public Health. 2009;9:298.

561 35. World Health Organization. mhGAP intervention guide for mental, neurological and substance

562 use disorders in non-specialized health settings: mental health Gap Action Programme (mhGAP): World 563 Health Organization; 2010.

564 36. Sokhela NE. The integration of comprehensive psychiatric/mental health care into the primary

565 health system: diagnosis and treatment. Journal of Advanced Nursing. 1999;30(1):229-37.

566 37. Jenkins R, Kiima D, Njenga F, Okonji M, Kingora J, Kathuku D, et al. Integration of mental health 567 into primary care in Kenya. World Psychiatry. 2010;9(2):118.

568 38. Kokota D, Lund C, Ahrens J, Breuer E, Gilfillan S. Evaluation of mhGAP training for primary 569 healthcare workers in Mulanje, Malawi: a quasi-experimental and time series study. International 570 Journal of Mental Health Systems. 2020;14(1):3.

571 39. Wright J, Common S, Kauye F, Chiwandira C. Integrating community mental health within 572 primary care in southern Malawi: a pilot educational intervention to enhance the role of health 573 surveillance assistants. Int J Soc Psychiatry. 2014;60(2):155-61.

574 40. Ventevogel P. Integration of mental health into primary healthcare in low-income countries:

575 avoiding medicalization. International Review of Psychiatry. 2014;26(6):669-79. 
576 41. Cataldo F, Kielmann K, Kielmann T, Mburu G, Musheke M. 'Deep down in their heart, they wish

577 they could be given some incentives': a qualitative study on the changing roles and relations of care

578 among home-based caregivers in Zambia. BMC health services research. 2015;15(1):1-10.

579 42. Keynejad RC, Dua T, Barbui C, Thornicroft G. WHO Mental Health Gap Action Programme

580 (mhGAP) Intervention Guide: a systematic review of evidence from low and middle-income countries.

581 Evidence-based mental health. 2018;21(1):30-4.

582 


\section{Supplementary Files}

This is a list of supplementary files associated with this preprint. Click to download.

- FGDsdatacollectiontool.pdf

- IDIsdatacollectiontool.pdf 